Technological University Dublin

DỨBLIN

ARROW@TU Dublin

2013-03-04

\title{
Omnidirectional Circularly Polarized Patch Antenna with Post Manufacture Characteristic Refinement
}

\author{
Adam Narbudowicz \\ Technological University Dublin \\ Xiulong Bao \\ Technological University Dublin, xiulong.bao@tudublin.ie \\ Max Ammann \\ Technological University Dublin, max.ammann@tudublin.ie
}

Follow this and additional works at: https://arrow.tudublin.ie/ahfrccon

\section{Recommended Citation}

Narbudowicz, A., Bao, X.L. \& Ammann, M.J. (2013) Omnidirectional Circularly Polarized Patch Antenna with Post Manufacture Characteristic Refinement. >i>International Workshop on Antenna Technology iWAT, Karlsruhe, Germany, pp. 31-34, 04/03/2013. doi:10.1109/IWAT.2013.6518291

This Conference Paper is brought to you for free and open access by the Antenna \& High Frequency Research Centre at ARROW@TU Dublin. It has been accepted for inclusion in Conference Papers by an authorized administrator of ARROW@TU Dublin. For more information, please contact arrow.admin@tudublin.ie, aisling.coyne@tudublin.ie,gerard.connolly@tudublin.ie. Funder: Science Foundation Ireland 


\title{
Omnidirectional Circularly Polarized Patch Antenna with Post Manufacture Characteristic Refinement
}

\author{
Adam Narbudowicz ${ }^{*(1)}$, Xiulong Bao ${ }^{(2)}$, and Max J. Ammann ${ }^{(3)}$ \\ Antenna \& High Frequency Research Centre, \\ Dublin Institute of Technology, Kevin Street, Dublin 8, Ireland \\ (1)adam.narbudowicz@mydit.ie \\ (2)xbao@dit.ie \\ (3)max.ammann@dit.ie
}

\begin{abstract}
We propose an omnidirectional circularly polarised planar microstrip patch antenna, in which both impedance bandwidth and circular polarization properties can be adjusted after antenna manufacture. This is done by appropriately tuning four adjustable capacitors, incorporated into the patches. To demonstrate the properties, the proposed antenna is tuned to the GPS L1 band and then re-tuned for Beidou/Galileo E2 band by only changing capacitors values. Design guidelines for such retuning are provided.
\end{abstract}

\section{INTRODUCTION}

In recent years there has been a growing interest in high performance circularly polarized (CP) antennas, due to many advantages it offers. Although basic CP antennas are widely known for a long time, it is only quite recently that compact designs offering ultra wide band [1] or omnidirectional performance [2] have been reported.

Traditionally omnidirectional CP radiation was achieved by employing an array of radiators, spaced concentrically around a common centre [3] or by adding polarisers around a linearly polarized antenna [4]. These approaches are not practical for some applications, as the resultant antennas are large in size and difficult to manufacture. Recently two other methods have been proposed, which allowed to implement omnidirectional CP performance with a planar structure. In [5] a metamaterial and a zeroth order resonance is used to produce omnidirectional CP in the plane of antenna's substrate. Circular polarisation is generated by employing four arm-shaped radiators for horizontal component and a zeroth order resonance of a patch in the middle for vertical component. Alternatively [2], [6], [7] employs two back-to-back coupled CP patches, which radiate omnidirectional CP in the plane orthogonal to the substrate. All above mentioned designs are compact, however operate within very narrow bandwidth and - although sufficient to cover GPS L1 band - it is difficult to manufacture the antenna on a commercial scale without the risk of frequency shift due to manufacturing inaccuracies. It is therefore important to develop a technique, enabling compensation for such inaccuracies after manufacture.

In this paper we propose a solution, by cutting slots in the patch of [6] and loading them with adjustable capacitors. This prolongs the current path of each radiating mode and by proper change of the capacitance one can adjust both impedance and axial ratio (AR) bandwidth.

\section{ANTENNA DESIGN}

The antenna consists of two back-to-back square patches with a common ground plane in the middle. A coplanar waveguide $(\mathrm{CPW})$ is placed diagonally and provides in-phase feed to both patches. Along the two top edges of each patch a slot is introduced and across the middle of each slot an adjustable capacitor is soldered. This prolongs the current path of each mode (please note, there are two orthogonal modes for each patch and so there are two orthogonal slots with capacitors), reducing the resonant frequency. It was previously demonstrated [7] that this shift in frequency is proportional i.a. to the lumped capacitance.

In order to achieve omnidirectional CP coverage with only two patches, a groundplane is substantially reduced (as compared to classical designs) to facilitate coupling between the two patches [6]. This reduces the antenna footprint, however for practical applications care has to be taken to avoid current flowing on the outside of the feed cable. 
The CPW couples in-phase energy into both patches. It is located diagonally in order to excite the two orthogonal resonant modes of each patch. At the bottom end of CPW there is a short $5 \mathrm{~mm}$ long section of microstrip line along with a CPW-to-microstrip transition employing two vias. This was done purely to facilitate the soldering of SMA connector.

In classical designs a perturbing element or a small variation between a width and length of the patch (so called "almost-square patch") would be introduced in order to achieve $90^{\circ}$ phase shift between the modes, required for CP. In proposed design all dimensions are symmetric and the phase shift is achieved by a small difference in the capacitance in the left $\left(C_{L}\right.$ on Fig. 1) and right $\left(C_{R}\right)$ slots. The resonant frequency of each mode is inversely proportional to the capacitor value, therefore such difference will produce the same effect as small difference between length and width of the patch. However, unlikely the classical approach, proposed antenna can be easily reconfigured from right hand (RHCP) to left hand CP (LHCP), or - if required - produce RHCP on the front side and LHCP on the back.

Fig. 1 shows the proposed design. The dimensions are: $L_{p}=48.5 \mathrm{~mm} ; S_{p}=1.9 \mathrm{~mm} ; L_{s}=25.4 \mathrm{~mm} ; W_{s}=1.4 \mathrm{~mm}$; $S=53.7 \mathrm{~mm} ; \Delta_{s}=3 \mathrm{~mm} ; \Delta_{f}=18.6 \mathrm{~mm} ; \Delta_{r}=2 \mathrm{~mm} ; L_{C P W}=46.4 \mathrm{~mm} ; L_{x}=37 \mathrm{~mm} ; W_{x}=20 \mathrm{~mm} ; L_{f}=12 \mathrm{~mm}$.

\section{RECONFIGURATION}

In the middle of each slot there is a capacitive load, which according to previous study should be around 1.5 $\mathrm{pF}$ [7]. This is much lower than the available on market adjustable capacitors, therefore the design was intended for a series connection of a fixed $2.2 \mathrm{pF}$ capacitor and an adjustable one with the $3-10 \mathrm{pF}$ range. This covers capacitances from 1.27 to $1.8 \mathrm{pF}$, which - for proposed antenna - result in resonant frequencies at 1.635 and $1.512 \mathrm{GHz}$ respectively (see Fig. 2). To include undesired losses of such connection, the capacitors were simulated with a serial resistance of $0.4 \Omega$.

For the initial design it is assumed that the resonant frequency is inversely proportional to the capacitors value. This allows to estimate the initial capacitance as:

$$
C_{d e s}=C_{B}+\left(C_{A}-C_{B}\right)\left(\frac{f_{B}-f_{d e s}}{f_{B}-f_{A}}\right)
$$

where:

$$
\begin{array}{ll}
f_{d e s} & \text { - desired resonant frequency } \\
C_{d e s} & \text { - desired capacitance } \\
f_{B} & \text { - highest tunable resonant frequency } \\
C_{B} & \text { - capacitance corresponding to } f_{B} \\
f_{A} & \text { - lowest tunable resonant freqency } \\
C_{A} & \text { - capacitance corresponding to } f_{A}
\end{array}
$$

The exact value for $\mathrm{C}_{\mathrm{des}}$ is optimized through full wave simulation in CST Microwave Studio. To achieve circular polarization a small variance in capacitance $\Delta \mathrm{C}$ is introduced, so that $\mathrm{C}_{\mathrm{L}}=\mathrm{C}_{\mathrm{des}}-\Delta \mathrm{C}$ and $\mathrm{C}_{\mathrm{R}}=\mathrm{C}_{\mathrm{des}}+\Delta \mathrm{C}$. Fig. 2 shows the effect of this perturbation on AR for the exemplary GPS L1 configuration. The increase of $\Delta \mathrm{C}$ yields better omnidirectional AR, however also increases VSWR. It was found, that - while tunning antenna at different frequencies - the lower frequencies required smaller $\Delta \mathrm{C}$ for good AR performance than the higher ones.

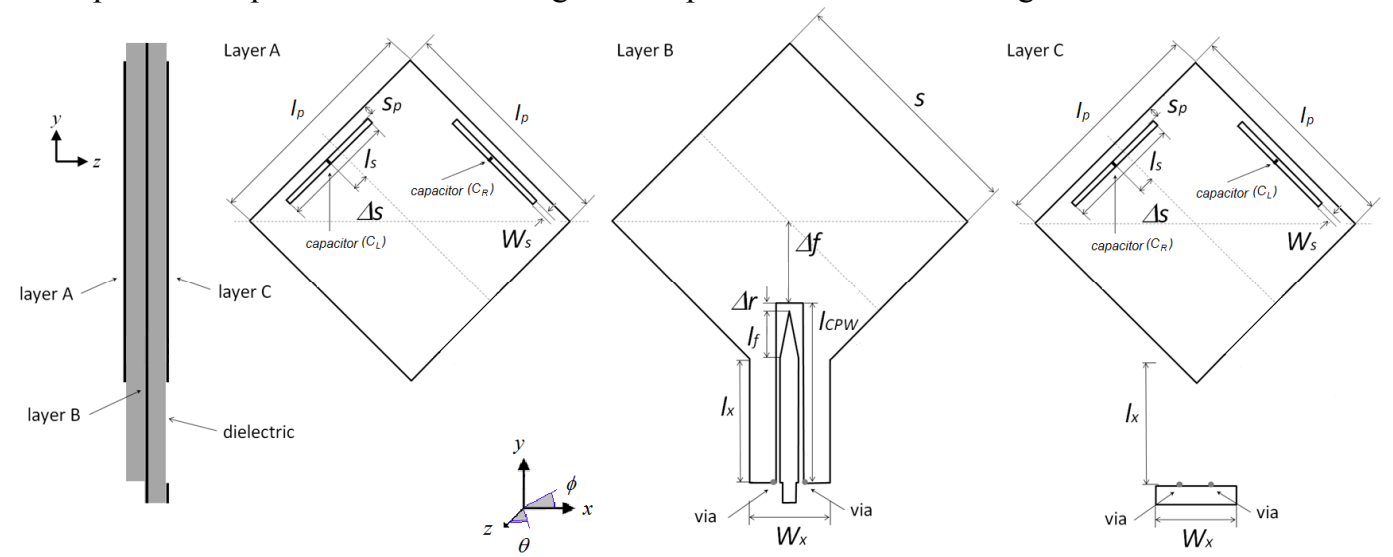

Fig. 1 Proposed design with each of three metalization layer shown (as seen along $z$ direction). Please note, that on layer $\mathrm{C}$ the capacitors marked as $C_{L}$ and $C_{R}$ are reversed as compared to layer A. 

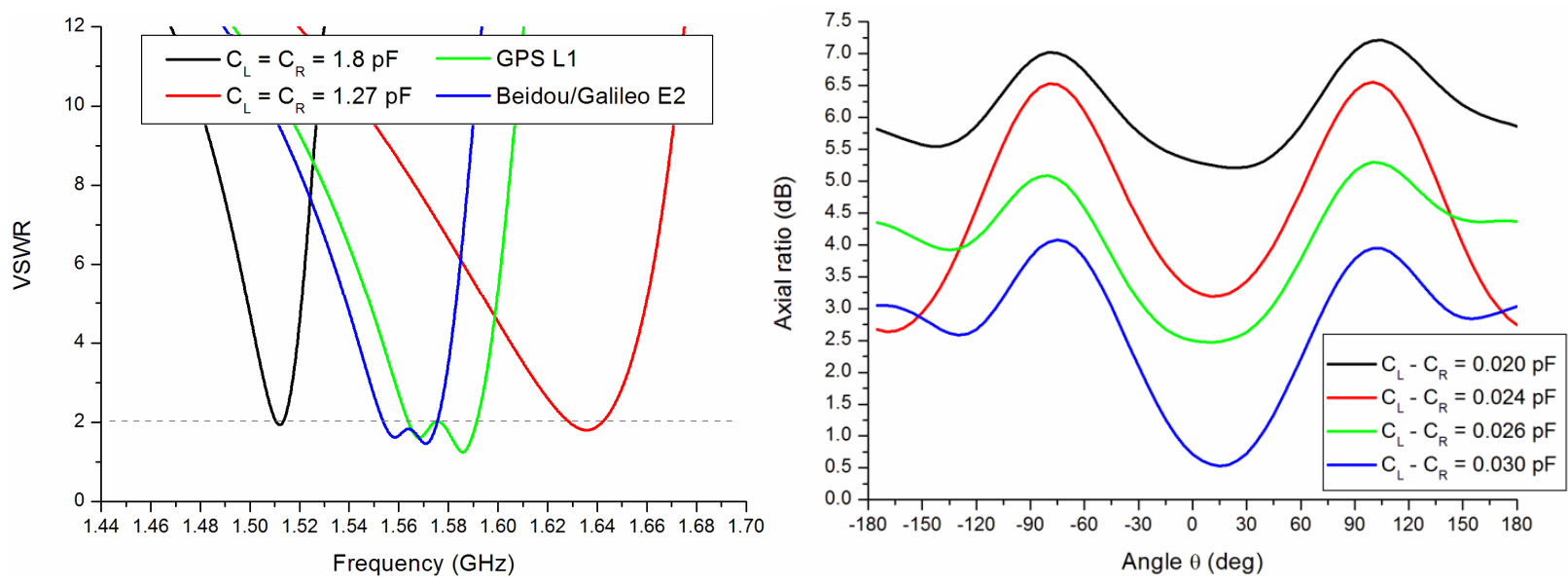

Fig. 2 Left: VSWR for the two presented configurations of proposed antenna and two configurations with minimum and maximum capacitances. Axial ratios for GPS L1 and Beidou/Galileo E2 configurations are shown on fig. 3. Right: Axial ratio in $z x$-plane for different values of $\Delta$ C, for GPS L1 configuration $1.575 \mathrm{GHz}$.
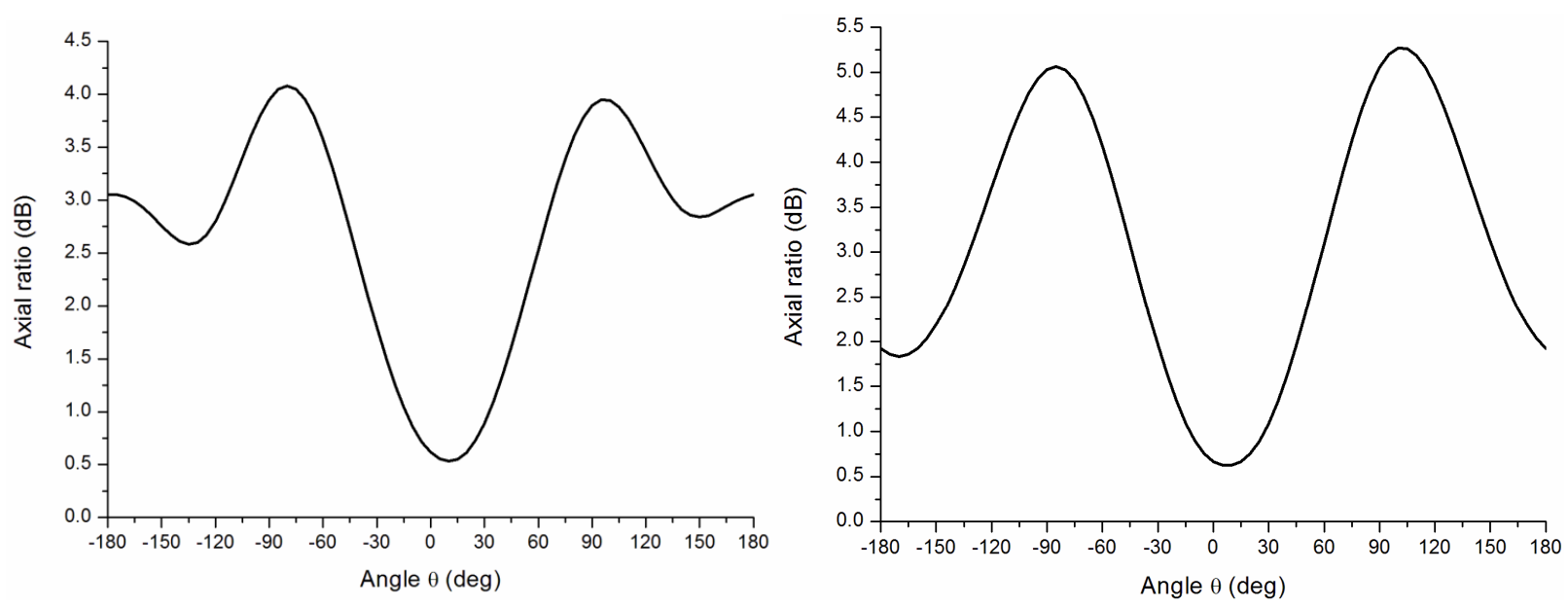

Fig. 3 Axial ratio for the GPS L1 (left) at $1.575 \mathrm{GHz}\left(\mathrm{C}_{\mathrm{L}}=1.549 \mathrm{pF} ; \mathrm{C}_{\mathrm{R}}=1.519 \mathrm{pF}\right)$ and Beidou / Galileo E2 configurations (right) at $1.561 \mathrm{GHz}$ $\left(\mathrm{C}_{\mathrm{L}}=1.623 \mathrm{pF} ; \mathrm{C}_{\mathrm{R}}=1.603 \mathrm{pF}\right)$

\section{GPS L1 Configuration}

The proposed technique is demonstrated here to tune the antenna to the GPS L1 band. Applying design parameters $\left(f_{\text {des }}=1.575 \mathrm{GHz} ; f_{A}=1.512 \mathrm{GHz} ; C_{A}=1.8 \mathrm{pF} ; f_{B}=1.635 \mathrm{GHz} ; C_{B}=1.27 \mathrm{pF}\right)$ into equation (1) yields $C_{\text {des }}=1.529 \mathrm{pF}$. In order to achieve omnidirectional $\mathrm{CP}$ pattern, a small difference between $\mathrm{C}_{\mathrm{L}}$ and $\mathrm{C}_{\mathrm{R}}$ was introduced and after some fine tuning (using CST Microwave Studio) resulting capacitances are $C_{L}=1.549 \mathrm{pF}$ and $C_{R}=1.519$. Simulated results show omnidirectional CP in $x z$-plane with worst axial ratio of $4.1 \mathrm{~dB}$ and a mean value of $2.64 \mathrm{~dB}$. It can be seen on the radiation plots, that the antenna behaves like a CP dipole, with an omnidirectional pattern in one plane and figureof-eight shape in the other (see Fig. 3-4). The asymmetry in the AR is due to the layer of prepreg glue, which was incorporated into the simulation.

\section{BEIDOU / GALILEO E2 Configuration}

To demonstrate the usefulness of proposed design, the antenna (keeping all physical dimensions the same) was tuned to the Beidou/Galileo E2 band which is centered at $1.561 \mathrm{GHz}$. Applying (1) yields initial capacitance of $1.589 \mathrm{pF}$, however during fine tuning (again with use of CST Microwave Studio) it was found that the optimum performance is achieved for slightly higher values. The reported configuration is $C_{L}=1.623 \mathrm{pF}$ and $\mathrm{C}_{\mathrm{R}}=1.603 \mathrm{pF}$.

Figs. 3 and 5 depict radiation patterns at $1.561 \mathrm{GHz}$. The AR in $x z$-plane is below $5.3 \mathrm{~dB}$ with mean value of $3.06 \mathrm{~dB}$. The radiation patterns are very similar to those in GPS L1 configuration. 

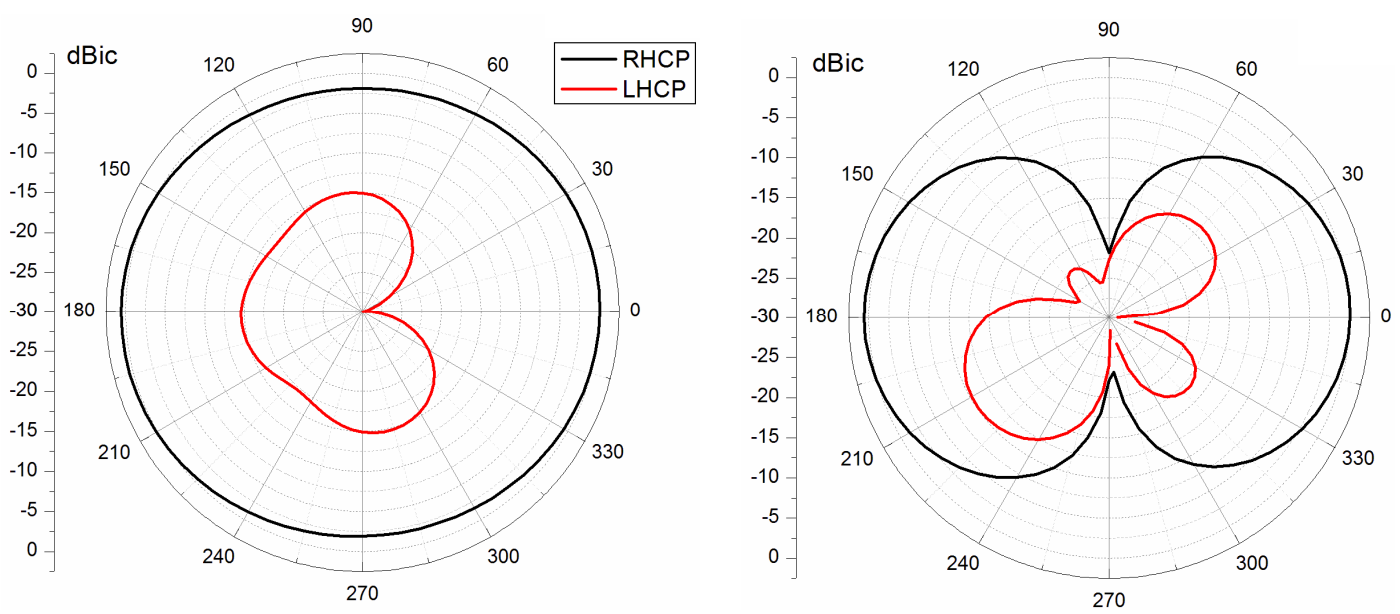

Fig. 4 Gains for GPS L1 configuration in $x z$-plane (left) and $y z$-plane (right) for $1.575 \mathrm{GHz}$.
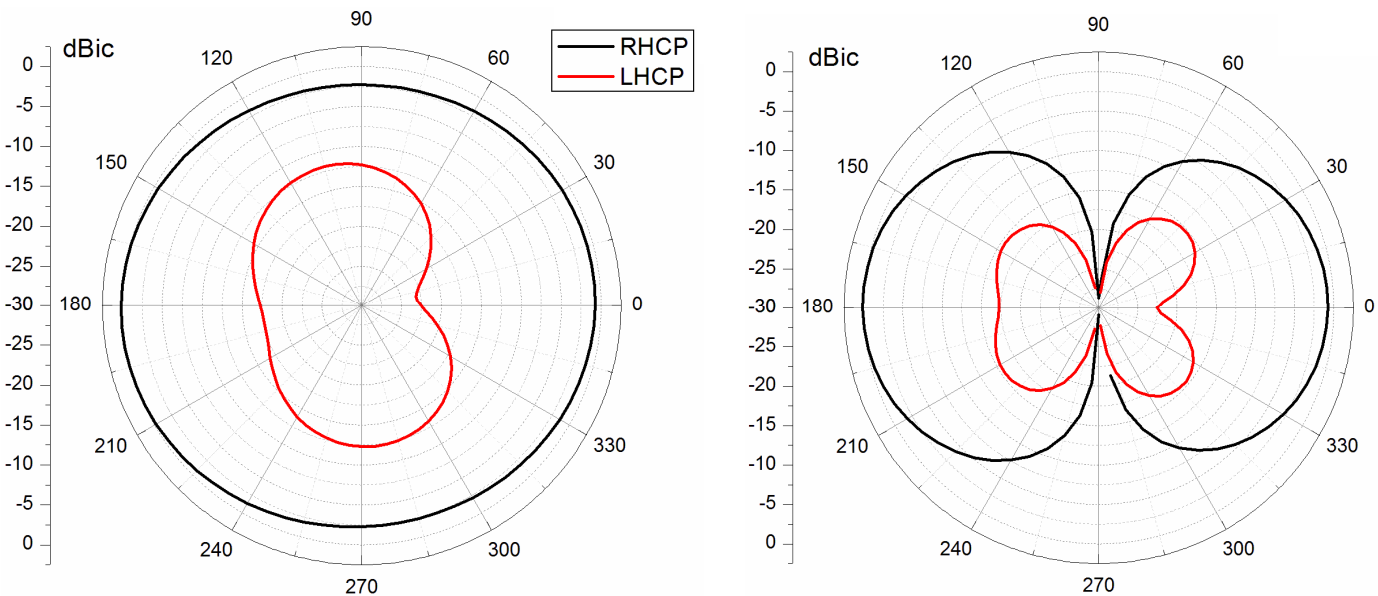

Fig. 5 Gains for Beidou / Galileo E2 configuration in $x z$-plane (left) and $y z$-plane (right) for $1.561 \mathrm{GHz}$.

\section{CONCLUSIONS}

In this paper we have presented a planar omnidirectional $\mathrm{CP}$ antenna, which can be adjusted to the desired band, $\mathrm{AR}$ level and sense of CP. As typically omnidirectional $\mathrm{CP}$ antennas are very narrowband $(\sim 5 \mathrm{MHz}$ of omnidirectional $\mathrm{CP}$ coverage), in a large scale production there is high risk of frequency shift due to manufacturing inaccuracies. The proposed design mitigates this problem. A similar methodology can be also applied to other types of narrowband microstrip patch antennas.

\section{REFERENCES}

[1] X.L. Bao, and M.J. Ammann, "Printed circularly polarised antenna with ultra-wide axial-ratio bandwidth," IET Microwaves, Antennas \& Propagation, vol. 5, pp. 1089-1096, June 2011.

[2] H. Iwasaki, and N. Chiba, "Circularly polarised back-to-back microstrip antenna with an omnidirectional pattern," IEE Proc. - Microwaves, Antennas \& Propagation, vol. 146, issue 4, pp. 277-281, Aug. 1999.

[3] R.C. Hall, and D.I. Wu, "Modeling and design of circularly-polarized cylindrical wraparound microstrip antennas," IEEE Ant. \& Prop. Symposium Digest, Baltimore, USA, 1996, vol. 1, pp. 672-675.

[4] Y. Pan, and K. Leung, "Wideband circularly polarized dielectric bird-nest antenna with conical radiation pattern," IEEE Transactions on Antennas and Propagation, in press.

[5] B.C. Park, and J.H. Lee, "Omnidirectional circularly polarized antenna utilizing zeroth-order resonance of epsilon negative transmission line," IEEE Trans. on Ant. and Prop., vol. 59, no. 7, pp. 2717-2721, Jul. 2011.

[6] A. Narbudowicz, X.L. Bao, and M.J. Ammann, "Omnidirectional circularly polarized microstrip patch antenna," Electronic letters, vol. 48, issue 11, pp. 614-615, May 2012.

[7] A. Narbudowicz, X.L. Bao, and M.J. Ammann, "Dual-band omnidirectional circularly polarized antenna," IEEE Transactions on Antennas and Propagation, in press. 